\title{
Robotic-assisted thoracoscopic resection of an anterior mediastinal vascular malformation
}

\author{
Georgios Geropoulos ${ }^{1}$, Konstantinos Kapriniotis ${ }^{1}$, Sofoklis Mitsos ${ }^{1}$, Emily Joslin², Michael Klimatsidas ${ }^{3}$, \\ Nikolaos Panagiotopoulos ${ }^{1}$
}

\begin{abstract}
${ }^{1}$ Thoracic Surgery Department, University College London Hospitals, NHS Foundation Trust, London, United Kingdom ${ }^{2}$ Cellular Pathology Department, Princess Alexandra Hospital, NHS Foundation Trust, Harlow, Essex, United Kingdom

${ }^{3}$ Department of Minimally Invasive Thoracic Surgery, St Luke's Hospital, Thessaloniki, Greece
\end{abstract}

Kardiochir Torakochir Pol 2020; 17 (4): 210-211

The mediastinum is defined as the space between the two lungs. Among the abnormal masses that have been described in the mediastinum, thymomas, teratomas and lymphomas are the most common ones. Vascular abnormalities are rarely reported in the mediastinum [1].

A 49-year-old female patient was admitted electively to the thoracic surgery department for management of an anterior mediastinal mass. The patient had a history of bilateral mastectomy 23 years ago for a breast lump. Following her regular follow-up, approximately 6 months ago a chest $X$-ray showed a shadow right of the sternum. Computed tomography (CT) showed a $48 \times 37 \times 35 \mathrm{~mm}$ superior anterior mediastinal mass just to the right of the midline and abutting the superior vena cava and the aortic arch, highlighting the lesion as highly suspicious for thymoma (Figure 1). A small central calcific focus was also noted. No other significant findings were described. A second CT scan showed that the findings were stable and a decision for a robotic assisted surgical excision was made. Under general anaesthesia and the patient in supine position slightly titled on

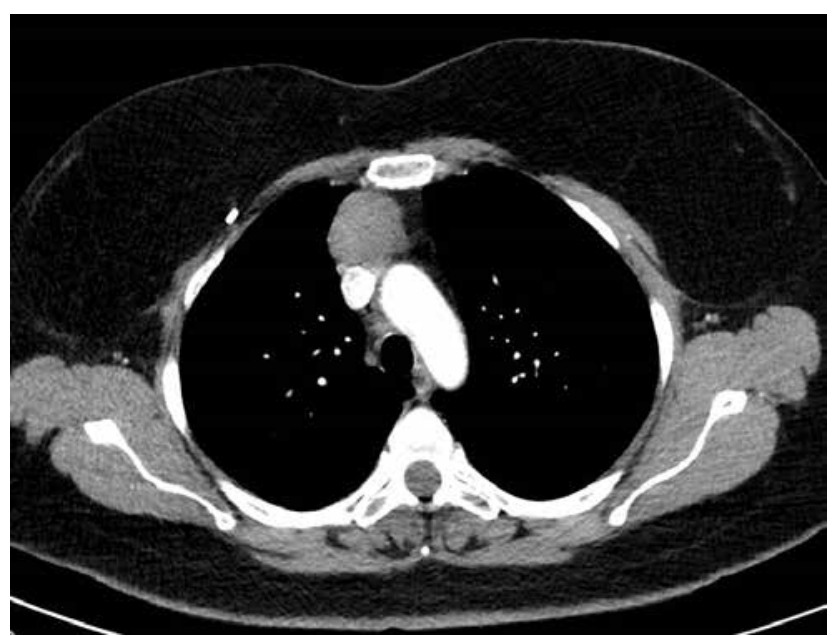

Figure 1. Computed tomography of the thorax. In the anterior mediastinum a mass anterior to the great vessels is identified the left a $12 \mathrm{~mm}$ camera port was inserted in the right anterior axillary line in the $5^{\text {th }}$ intercostal space. $\mathrm{CO}_{2}$ insufflation was done. A second $8 \mathrm{~mm}$ port was inserted parasternally for the monopolar incisors. Another $8 \mathrm{~mm}$ port was inserted under the axilla 5-6 cm anteriorly for the bipolar grasper. Finally an assisting $12 \mathrm{~mm}$ port was inserted onto the $10^{\text {th }}$ intercostal space anterior to the mid axillary line for the suction-irrigation device and the introduction of swabs. The lesion was identified as per CT findings and it was extremely vascular on its surface. As there was a vascular communication between the mass itself, the superior vena cava and the innominate vein, the neck of the mass was transected with an endoscopic vascular stapler. The mass was removed with an EndoBag. Careful haemostasis was performed and a 28 French drain was inserted. The lung was fully expanded, and the patient was discharged on the first postoperative day. Histological examination confirmed that the lesion was a vascular malformation (Figure 2).

Vascular abnormalities are rarely seen in the mediastinum and their incidence is thought to approximate less than $0.5 \%$ of mediastinal masses [2]. From a histological point of view, cavernous and capillary haemangiomas account for more than $90 \%$ of mediastinal abnormalities and in general only $0.8 \%$ of haemangiomas have an extra-cutaneous location [2, 3].

As described, in the majority of the cases the definite treatment is surgical resection when technically applicable. There is no evidence regarding surgical resection as most of them are asymptomatic lesions. However, it is worth mentioning that in rare cases the lesion if left untreated could be symptomatic and unresectable [4]. Furthermore, in some case the preoperative embolization may reduce the blood flow and thus reduce the volume of the vascular malformation. This may increase the surgical resectability of the mass [5].

On the other hand, preoperative computed tomography may be suggestive for a cystic lesion and thus be non-diagnostic [6]. In such cases, a transthoracic ultrasound Doppler may be useful. Cheng et al., in their case, following an

Address for correspondence: Georgios Geropoulos MD, Thoracic Surgery Department, University College London Hospitals, NHS Foundation Trust, London, UK, e-mail: georgios.geropoulos@nhs.net

Received: 6.08.2020, accepted: 24.10.2020. 

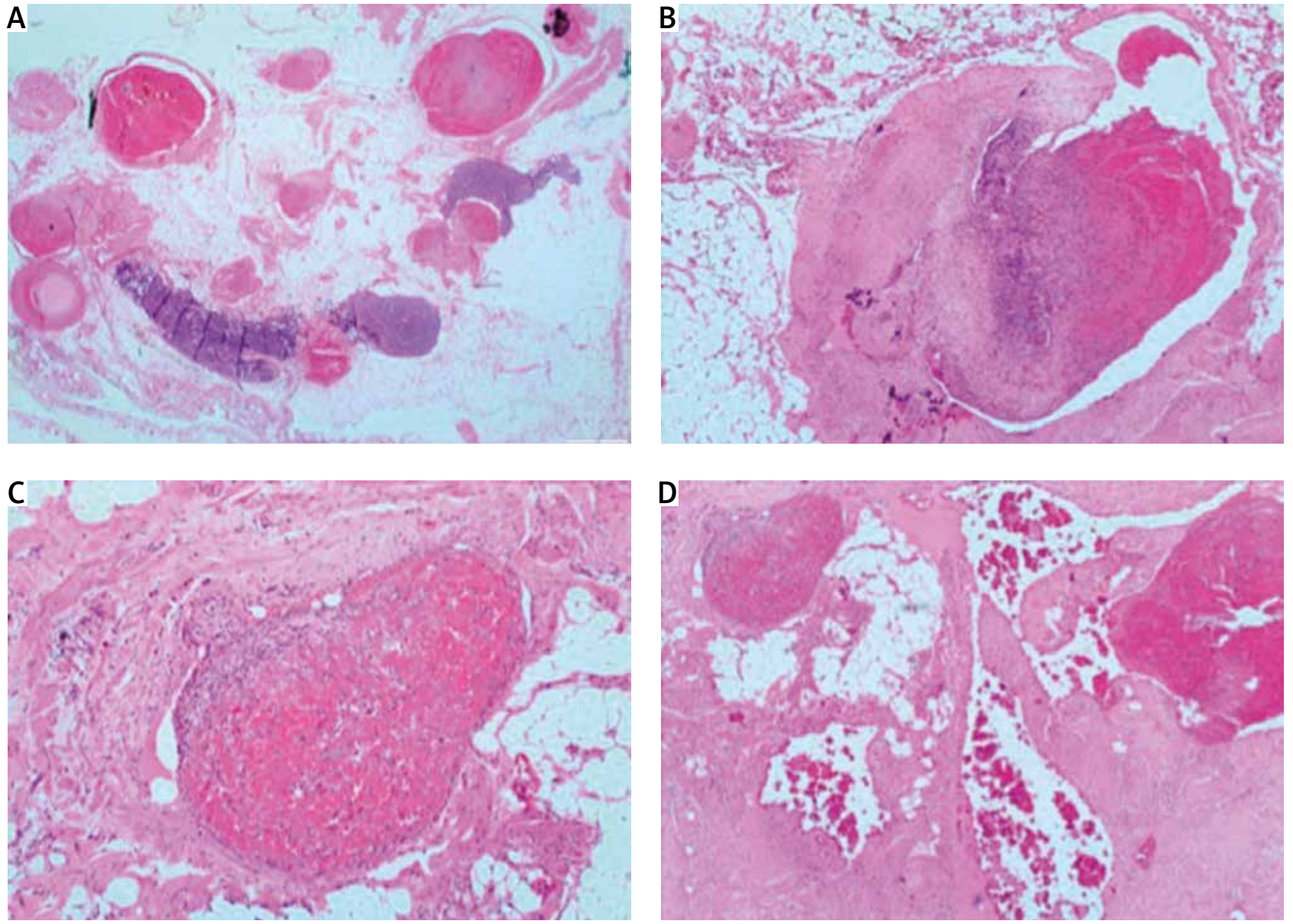

Figure 2. Arteriovenous malformation within the mediastinum showing dilated thick and thin walled vessels with an intervening lymphoid stroma, with evidence of organising thrombus. A - Hematoxylin and eosin stain (H\&E) representative 1.25×. B - H\&E representative $4 \times$. C - H\&E representative 10x. D - H\&E representative 40x

abnormal chest X-ray of their patient, were able to confirm the vascular origin of the mediastinal mass performing a transthoracic Doppler ultrasound [7].

In conclusion, most mediastinal vascular malformations are asymptomatic. When a mediastinal mass is highly suspicious to have a vascular origin, a needle biopsy should be postponed and surgical resection has to be considered for a definite diagnosis. A minimal invasive surgical approach can be a safe option for management of mediastinal vascular malformations. However, careful dissection and ligation of the vascular supply of the vascular malformation is of increased importance, in order to completely resect the mass and avoid perioperative bleeding.

\section{Disclosure}

The authors report no conflict of interest.

\section{References}

1. Carter BW, Marom EM, Detterbeck FC. Approaching the patient with an anterior mediastinal mass: a guide for clinicians. J Thorac Oncol 2014; 9: S102S109.

2. McAdams HP, Rosado-de-Christenson ML, Moran CA. Mediastinal hemangioma: radiographic and CT features in 14 patients. Radiology 1994; 193: 399-402.

3. Mizutani E, Morita R, Kitamura S. Arteriovenous hemangioma in the middle mediastinum: report of a case. Surg Today 2011; 41: 846-848.

4. Grillo HC, Athanasoulis CA. Tracheal obstruction from mediastinal arteriovenous malformation. J Thorac Cardiovasc Surg 2004; 128: 780-782.

5. Li J, Liu H, Ye L. Coil embolization for a vast and complex arteriovenous malformation in the posterior mediastinum. Int I Clin Exp Med 2015; 8: 16838-16841.

6. Kaplan T, Altuntaş B, Ceran S, Sunam GS. Unusual location of arteriovenous malformation; posterior mediastinum. Interact Cardiovasc Thorac Surg 2009, 8: 260-262.

7. Cheng SL, Wang HC, Yang PC. Posterior mediastinal arteriovenous malformation with atypical color Doppler sonographic findings. J Clin Ultrasound 2003; 31: 44-47. 